\title{
CONHECIMENTO SOBRE TABAGISMO ENTRE OS ACADÊMICOS DAÁREA BIOMÉDICA DA UNIVERSIDADE FEDERAL DO ESPÍRITO SANTO
}

\author{
Cremilda Maria de Mello Silva ${ }^{1}$, Márcia Bárbara Souza Dibai², Nágela Valadão Cade ${ }^{3}$
}

\begin{abstract}
RESUMO: Foi realizado um estudo comparativo com o objetivo de conhecer o nível de informação sobre tabagismo entre os alunos dos períodos iniciais e finais dos cursos de graduação da área biomédica da Universidade Federal do Espírito Santo. Os dados foram coletados em fevereiro de 2004 por meio de questionário e analisados estatisticamente. Os resultados mostraram um percentual mediano de $66,7 \%$ de acerto das respostas e os alunos do curso de medicina obtiveram $71 \%$ de acerto $(p<0,01)$ em relação aos demais cursos. A média de acertos entre os períodos iniciais e finais de todos os cursos analisados revelou uma diferença significante $(\mathrm{p}<0,05)$, sendo maior entre os alunos dos períodos finais. O nível de acertos foi mais elevado entre os alunos que tiveram o tema abordado em maior número de disciplinas.
\end{abstract}

PALAVRAS-CHAVE: Tabagismo; Conhecimento; Estudantes.

\section{KNOWLEDGE ON SMOKING AMONG BIOMEDICAL UNDERGRADUATES AT THE FEDERAL UNIVERSITY OF ESPÍRITO SANTO}

\begin{abstract}
A comparative study was carried out objectifying to learn the level of information on smoking among students in the initial and final terms of biomedical graduation courses at the Federal University of Espírito Santo. Data were collected in February 2004 by means of a questionnaire and statistically analysed. The results showed a median percentage of $66.7 \%$ correct answers, and medical students obtained a correctness rate of $71 \%(\mathrm{p}<0.01)$ in relation to other courses. Average correct answers among the undergraduates in the initial and final terms disclosed a significant difference $(\mathrm{p}<0.05)$, being higher among final-term students. The number of correct answers was higher among students who had the subject approached in more disciplines.
\end{abstract}

KEYWORDS: Smoking; Knowledge; Students.

\section{CONOCIMIENTO SOBRE TABAQUISMO ENTRE LOS ALUMNOS DEL ÁREADE LA SALUD DE LA UNIVERSIDAD FEDERAL DE ESPÍRITO SANTO}

RESUMEN: Fue hecho un estudio comparativo con el objetivo de conocer el nivel de información sobre tabaquismo entre los alumnos de los periodos iniciales y finales de los cursos de graduación del área de la salud de Universidad Federal de Espírito Santo. Los datos fueron recogidos en febrero de 2004 por entrevista estructurada y analizados con métodos de estadística. Los resultados mostraron un porcentual mediano de 66,7\% de aciertos de las respuestas y los alumnos del curso de medicina han obtenido $71 \%$ de acierto $(\mathrm{p}<0,01)$ en relación a los demás alumnos de otros cursos. La media de aciertos entre los periodos iniciales y finales de todos los cursos analizados reveló una diferencia significante $(\mathrm{p}<0,05)$, siendo mayor entre los alumnos de los periodos finales. El nivel de aciertos fue entre los alumnos que tuvieron el tema tabaquismo en mayor número de disciplinas.

PALABRAS CLAVE: Tabaquismo; Conocimiento; Estudiantes.

${ }^{1}$ Enfermeira. Coordenadora do Programa de Tabagismo da Secretaria Estadual de Saúde/ES. Mestre em Saúde Coletiva pela Universidade Federal do Espírito Santo-UFES.

${ }^{2}$ Assistente Social. Hospital Universitário Cassiano Antônio de Moraes/ES. Mestre em Saúde Coletiva pela UFES.

${ }^{3}$ Professora. Doutora. Departamento de Enfermagem e Programa de Pós-Graduação em Atenção à Saúde Coletiva da UFES.

Autor correspondente:

Nágela Valadão Cade

Av. Carlos Orlando de Carvalho 781/204 - 29260-060 - Vitória-ES.

Recebido: 30/06/08

E-mail:nagelavc@terra.com.br

Aprovado: 20/10/08

Cogitare Enferm 2008 Out/Dez; 13(4):542-7 


\section{INTRODUÇÃO}

No Brasil, foi a partir da década de 70 que se tornaram mais evidentes as manifestações organizadas para o controle do tabagismo. Na esteira das publicações científicas e de divulgação na mídia leiga, surgiram os primeiros projetos de lei, as manifestações públicas, as declarações de entidades e algumas leis municipais e estaduais restritivas, bem como as campanhas e programas de educação em saúde, principalmente dirigidas a escolares voltados para o controle do tabagismo.

Durante nove anos, as ações educativas foram apenas pontuais - nas duas datas anuais de realização das campanhas. A partir de 1996 passaram a envolver também ações contínuas - programadas ocorrendo ao longo de todo o ano. $\mathrm{O}$ alcance nacional dessas ações se deu graças a um processo de descentralização através da parceria com secretarias estaduais e municipais de saúde e com Organizações não Governamentais (ONGS). Esse processo contribuiu para uma significativa mudança de paradigma, no qual o tabagismo, de um comportamento elegante e charmoso, vem se transformando progressivamente num comportamento indesejável.

Em 1988, o Ministério da Saúde dos Estados Unidos publicou um amplo relatório sobre estudos que comprovam a capacidade do tabaco de causar dependência, o que levou a comunidade científica mundial a reconhecer o tabagismo como uma dependência química ${ }^{(1)}$. No entanto, apesar de todas as evidências apresentadas no meio acadêmicocientífico, em razão da expansão da epidemia do tabagismo, o processo de transformar esse conhecimento em ações e estratégias efetivas para o controle do tabagismo, ocorre lentamente. Tal situação possivelmente decorre do fato de que essas ações envolvem diferentes aspectos - sanitários, ambientais, econômicos, sociais e políticos - e estratégias.

A fim de controlar as conseqüências decorrentes do tabagismo, estratégias internacionais e nacionais têm sido elaboradas, englobando as diferentes fases do problema que compreende basicamente a produção do tabaco, o consumo de seus derivados, os efeitos nocivos no organismo, as medidas de prevenção da iniciação e o tratamento dos tabagistas. Quanto ao tratamento, a estratégia recomendada é a de que todos os tabagistas devem ser abordados e aconselhados a deixar de fumar por profissionais de saúde ${ }^{(2)}$.

No entanto, apesar de haver a preocupação e o reconhecimento do Ministério da Saúde (MS) e do Instituto Nacional de Câncer (INCA) em abordar e tratar o fumante, essa idéia vem sendo gradativamente incorporada entre esses profissionais, sendo citadas algumas barreiras para a sua efetivação, como por exemplo: As informações sobre como aconselhar e apoiar o paciente e sobre os métodos para deixar de fumar, por serem relativamente recentes, ainda não são amplamente conhecidos pela maioria dos profissionais de saúde ${ }^{(3)}$; Por outro lado ainda não existe interesse suficiente na maioria dos profissionais de saúde para atender tal clientela, mesmo porque muitos destes são fumantes ${ }^{(4)}$; Ainda predominam no país as ações assistenciais apesar das propostas de ações integrais propostas pelo SUS (Sistema Único de Saúde) que contrapõem as medidas de controle e prevenção, dentre as quais as relacionadas ao tabagismo ${ }^{(5)}$.

Um estudo multicêntrico, internacional envolvendo 9000 estudantes de medicina, de 51 escolas, oriundos de 42 países, identificou que apesar da maioria desses acadêmicos reconhecerem de um modo geral, que o fumo é extremamente prejudicial à saúde, existe acentuado desconhecimento sobre as conseqüências do fumo em determinadas áreas. Os achados mostraram que apenas 29\% dos estudantes do último ano de medicina na Europa, 32\% nos EUA e 43\% na Austrália sabem que o fumo é a principal causa de doenças vasculares.

Outro aspecto apontado por esse estudo é o de que, "na maioria dos países estudados, apenas 30\% a 49\% dos estudantes disseram que aconselhariam o paciente a abandonar o fumo, sendo que, no Japão, esse percentual foi de apenas 5\%”. Uma curiosidade nesse estudo é que os percentuais encontrados foram sempre menores quando os estudantes eram fumantes ${ }^{(6)}$.

A Organização Mundial da Saúde (OMS), em reuniões que realiza periodicamente desde 1999, com os 192 representantes de seus países membros a fim de tratar dos problemas decorrentes do tabagismo, vem inserindo no bojo de recomendações as relacionadas com abordagem e tratamento dos fumantes. Destaca a relevância do preparo do profissional de saúde em realizar abordagens e intervenções aos pacientes tabagistas de maneira a encorajá-los ao alcance e manutenção da abstinência 
de uso do tabaco. Na reunião de 1999 que teve por objetivo o delineamento de políticas européias para cessação do fumar, o foco foi a importância de todos os pacientes serem indagados sobre o tabagismo em todas os atendimentos de saúde e aconselhados a deixar de fumar ${ }^{(7)}$.

Diante do exposto consideramos importante despertar o interesse dos alunos, em particular os da área de saúde sobre esse campo de conhecimento. Uma vez que esses serão os profissionais a atuarem na prática e, possivelmente, a deparar-se com pacientes tabaco dependentes, necessitando de abordagem para que deixem de fumar.

Diante da hipótese de que alunos da área de saúde, ao final do curso de graduação, apresentam mais informações sobre tabagismo em relação aos alunos do primeiro período e que essas informações têm relação com o número de disciplinas que abordam essa temática ao longo da graduação, este estudo objetivou averiguar o nível de conhecimento entre os acadêmicos da área biomédica - Enfermagem, Farmácia, Medicina e Odontologia - no início e do final dos cursos.

\section{MÉTODO}

Foi realizado um estudo comparativo, com abordagem quantitativa, em um hospital público federal, sendo incluídos os alunos dos períodos iniciais e finais de quatro cursos de graduação na área da saúde - Enfermagem, Farmácia, Medicina, Odontologia.

A amostra do estudo compôs-se de dois grupos de alunos, classificados como grupo A (totalizando 94 alunos dos períodos iniciais dos respectivos cursos) e grupo $\mathrm{B}$ (totalizando 80 alunos dos últimos períodos dos mesmos cursos). Totalizando os dois grupos (A e B), a distribuição de alunos por cursos compunhase da seguinte forma: 42 alunos de enfermagem, 26 de farmácia, 60 de medicina e 46 de odontologia, sendo que ao todo 174 alunos responderam o questionário, assim em todos os cursos houve o percentual acima de $50 \%$ do total de alunos distribuídos por turma.

Os dados da pesquisa foram coletados em fevereiro de 2004 através de um questionário estruturado aplicado aos participantes da pesquisa. Anteriormente, foi aplicado um teste piloto a cinco estudantes escolhidos aleatoriamente a fim de avaliar o instrumento da pesquisa.
O questionário continha 21 questões que abordavam aspectos relativos ao tabagismo e as perguntas foram elaboradas com base em materiais do Ministério da Saúde (MS) e do Instituto Nacional do Câncer (INCA) que são veiculadas em instituições de saúde da rede do SUS e em eventos de capacitação profissional na área da saúde e da educação.

Foi avaliado o conhecimento geral sobre tabagismo com as questões: o tabagismo como fator de risco para várias doenças e como causa direta de adoecimento; da legislação brasileira sobre a temática; dos riscos e agravos relacionados ao consumo; riscos do fumo passivo; agravos ao meioambiente; abordagem e tratamento; e estatísticas de estudos prévios. Optou-se por computar os erros e acertos de cada questão isoladamente, sem emitir pontuação das respostas. Contemplou-se, em uma pergunta, se o aluno havia recebido informação específica sobre tabagismo em alguma disciplina e, caso afirmativo, solicitou-se que anotassem as referidas disciplinas que abordavam o tema.

Os questionários foram aplicados em sala de aula por um dos pesquisadores $(60.9 \%)$ e pelo professor da turma (39\%). Os dados obtidos foram tabulados na planilha do Programa Microsoft Excel e, posteriormente, analisados estatisticamente por meio do programa Statistical Package for the Social Sciences (SPSS).

Foi utilizado o teste qui-quadrado na comparação de acertos nas respostas dos alunos que cursavam períodos iniciais e daqueles que cursavam os períodos finais e o teste Post Hoc de Tukey para analisar diferenças entre os acertos dos alunos dos quatro cursos.

A fim de cumprir com os preceitos éticos, foi solicitada a todos os coordenadores dos cursos autorização para que a pesquisa fosse realizada na instituição, tendo havido o consentimento.

Os alunos foram, previamente esclarecidos, quanto aos objetivos da pesquisa, o tipo de colaboração que se desejava receber, o modo como os dados seriam registrados e utilizados para publicação, bem como foi especificada a liberdade que teriam em recusar a participar do estudo. Aos que concordaram em participar da pesquisa aplicou-se o Termo de Consentimento Livre e Esclarecido. Na época, os autores entenderam que as preocupações éticas referiam somente ao Termo de Consentimento Livre e Esclarecido porque se tratava de um estudo para 
avaliar conhecimento, sem envolvimento dos participantes à intervenção de riscos. A fim de manter o direito ao anonimato e sigilo acerca das respostas obtidas procedeu-se pela não identificação dos sujeitos no instrumento de pesquisa.

\section{RESULTADOS}

Houve uma variação etária de 18 a 28 anos, sendo $37,9 \%$ de estudantes do sexo masculino, $57,5 \%$ de estudantes do sexo feminino e $4,6 \%$ não assinalaram a qual gênero pertencia.

Ocorreu predomínio de alunos que se classificaram como sendo de cor branca $(50,6 \%)$, seguida daqueles de cor negra (2,9\%) e 37,4\% não responderam.

Quanto à religião $64,4 \%$ dos alunos eram católicos, 20,1\% evangélicos, $8 \%$ espíritas, 0,6\% declararam ter outra religião, $6,3 \%$ não tinham religião e $0,6 \%$ não responderam.

Quanto à renda familiar 50\% dos entrevistados declararam que a família ganhava mais de 10 salários mínimos, 29,3\% declararam a renda de 8 a 10 salários mínimos, 15,5\% declararam renda de 4 a 7 salários mínimos e 2,3\% declararam receber até 2 salários e não responderam 2,9\% dos entrevistados.

Quanto ao status em relação ao hábito de fumar, os estudantes se classificaram em sua maioria como nunca fumantes (91,3\%), seguidos daqueles que se consideravam fumantes $(4,0 \%)$ e os exfumantes (3,4\%). Não responderam 1,1\% dos alunos.

Em análise descritiva, considerando todos os cursos, houve um percentual de $66,7 \%$ de acerto das respostas e os alunos do curso de medicina obtiveram mais acertos (71\%), em relação aos alunos dos demais cursos. A média de acertos entre os períodos iniciais (grupo A) e finais (grupo B) dos cursos analisados revelou uma diferença significante $(\mathrm{p}<0,05)$ do conhecimento, nos períodos finais (Figura 1).

Comparando o total de acertos entre os cursos, os alunos de Medicina foram os que apresentaram maior percentual (Figura 2) e ainda o curso que teve o maior número de disciplinas que abordava sobre $o$ tabagismo (Tabela 1). Entretanto, apenas 26,6\% deles citaram as referidas disciplinas que abordaram sobre a temática em questão (Tabela 2).

Um fato surpreendente foi que entre os alunos do curso de farmácia não houve citação de nenhuma disciplina que tenha abordado o tema tabagismo. Porém, na análise geral, em relação aos demais cursos, o percentual de acertos desses alunos está equiparado aos alunos dos cursos de enfermagem e de odontologia (Figura 2).

Tabela 1 - Percentual médio e mediano de acertos

\begin{tabular}{lcccc}
\hline \multicolumn{1}{c}{ Grupo } & Média & Desvio & Mediana & Signif. \\
\hline Período & & & & \\
Inicias & 65,0 & 8,9 & 66,7 & $<0,05$ \\
Finais & 68,9 & 10,9 & 71,4 & \\
Disciplina ofertada & & & & \\
Sim & 66,9 & 10,3 & 66,7 & 0,406 \\
Não & 68,6 & 9,9 & 66,7 & \\
Curso & & & & \\
Enfermagem & 64,1 & 9,5 & 66,7 & \\
Farmácia & 66,5 & 7,4 & 66,7 & $<0,01$ \\
Medicina1 & 70,8 & 9,3 & 71,4 & \\
Odontologia & 64,4 & 11,4 & 66,7 & \\
\hline
\end{tabular}

1 - $\mathrm{O}$ teste Post Hoc de Tukey mostrou diferença estatística de medicina em relação aos outros 3 cursos

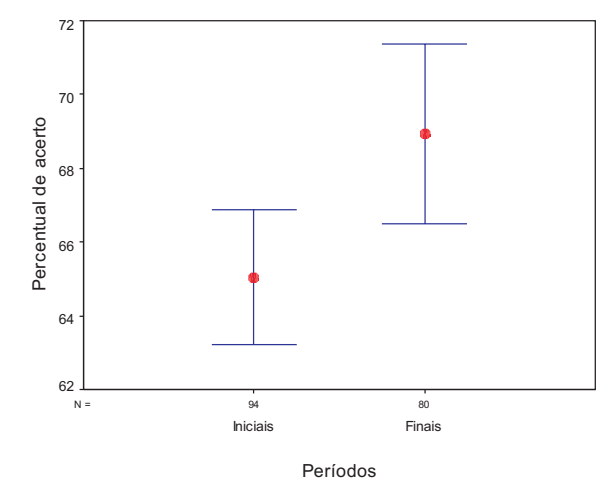

Figura 1 - Intervalo de confiança para a média do percentual de acertos entre os períodos iniciais e finais

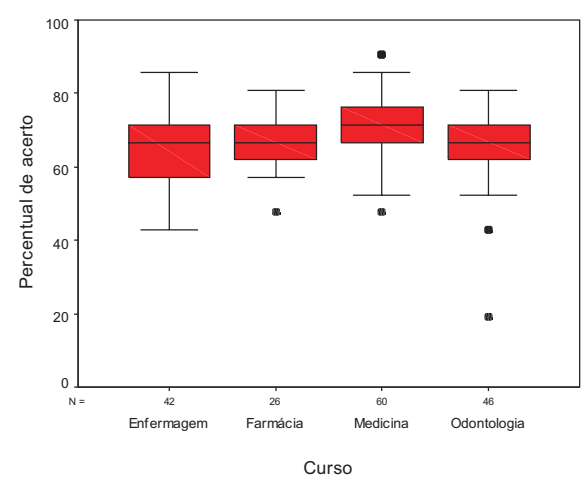

Figura 2. Distribuição do percentual de acerto entre os cursos 
Tabela 2 - Percentual de alunos que citou ter recebido informações sobre tabagismo e as disciplinas citadas por eles que abordaram o tema tabagismo

\begin{tabular}{cccl}
\hline Curso & $\begin{array}{c}\text { Total de } \\
\text { alunos }\end{array}$ & $\begin{array}{c}\text { \% de alunos } \\
\text { que citaram a } \\
\text { disciplina }\end{array}$ & Disciplinas citadas \\
\hline Enfermagem & 42 & $7,14 \%$ & Saúde Mental \\
Farmácia & 26 & $0,0 \%$ & Não houve citação \\
Medicina & 60 & $26,6 \%$ & Embriologia, \\
& & & Anatomia, \\
& & Patologia, \\
& & Pneumologia, \\
& & & Clínica Médica, \\
& & Oncologia, \\
& & & Epidemiologia e \\
& & Clínica Cirúrgica \\
& & & Formação Social, \\
& & Anatomia e \\
& & & Patologia Oral \\
\hline
\end{tabular}

\section{DISCUSSÃO}

Uma síntese dos resultados do estudo mostrou que ocorreu uma baixa prevalência do tabagismo entre os alunos pesquisados, corroborando com resultados de pesquisas realizadas na área que têm demonstrado a redução da prevalência do tabagismo nos países que programam ações de controle ${ }^{(8)}$. Por ser o tabagismo atualmente considerado como um problema de saúde pública, esforços vêm sendo somados, nacional e internacional, para reverter o aumento do número de fumantes, o número de doenças tabaco relacionadas, número de óbitos e os altos investimentos em tratamento.

$\mathrm{O}$ percentual de acertos foi maior entre todos os alunos dos períodos finais de todos os cursos. E está relativamente relacionado à ocorrência de alguma disciplina que tenha abordado sobre a temática.

Dessa forma, pôde ser constatado um percentual de acertos proporcionalmente maior entre os alunos do curso no qual havia a oferta de um maior número de disciplinas abordando sobre o tema, como ocorreu entre os alunos do curso de medicina que citaram um total de oito disciplinas que trataram da temática.

Contudo, os alunos dos demais cursos, que não tiveram nenhuma disciplina abordando o tema ou que tiveram até três disciplinas apenas, apresentaram um percentual de acerto equiparado. Aventamos que tal resultado está relacionado à obtenção do conhecimento através de outras fontes de informação além daquelas obtidas por meio do ensino acadêmico, contribuindo para a construção desse conhecimento, do senso comum.

Atualmente, as informações sobre tabagismo são veiculadas de várias maneiras na sociedade, como por exemplo, reportagens em revistas e jornais, campanhas educativas em instituições de saúde e escolas (com distribuição de folderes, cartazes) e outras iniciativas. A partir da década de 80 há uma tendência histórica de valorização do tabagismo na prática clínica passando a ser reconhecido não somente como fator de risco de doença e sim como uma condição clínica. E a partir de então passa a ser configurado na literatura médica não apenas como citação, mas no formato de capítulos e sub-capítulos ${ }^{(9)}$.

Dentre as ações de controle do tabagismo desenvolvidas no Brasil, as de prevenção são a que mais se revela na relação custo-benefício de uma forma geral, para as partes envolvidas. A medida de maior eficácia apontada pelo Ministério da Saúde (MS) e pelo Instituto Nacional do Câncer (INCA) seria justamente a abordagem dos tabagistas realizada por profissionais de saúde em seus atendimentos de rotina para a cessação do fumar. Pesquisas demonstram que, se todos os profissionais de saúde estiverem comprometidos com a prevenção e controle do tabagismo, as chances de sucesso serão maiores.

Segundo Rosemberg ${ }^{(10)}$, mesmo nos Estados Unidos, a falta de conhecimentos adequados e inabilidade para o aconselhamento médico voltado a obter a cessação do hábito de fumar é um obstáculo importante a ser vencido. Quase 90\% dos médicos clínicos consultados naquele país relatam que é necessário maior aprendizado, refletido num enriquecimento dos currículos das escolas médicas, para abordar o fumante na cessação do tabagismo.

Considerando que vários estudos têm demonstrado que os métodos para tratamento, assim como o tipo de abordagem a ser feita com o fumante ainda é desconhecido, comprometendo, dessa forma, a política da prevenção, é de suma importância o incentivo à capacitação entre todos os profissionais, particularmente os da área da saúde, uma vez que eles irão se deparar constantemente com essa problemática no seu cotidiano profissional ${ }^{(11-12)}$.

\section{CONCLUSÃO}

O conhecimento dos alunos a respeito do tabagismo e suas conseqüências se mostrou relacionado ao fato de a temática ter sido abordada durante sua 
formação acadêmica. Outras fontes de informação se mostraram como propulsoras para a disseminação de conhecimento nessa área, revelando o resultado das ações para o controle do tabagismo no Brasil.

Os alunos do Curso de Medicina têm tido mais oportunidade de adquirir esse conhecimento uma vez que o curso oferece em sua grade curricular diversas disciplinas que abordam o tema, em detrimentos aos alunos dos demais cursos da área da saúde. Essa realidade não favorece que todos os profissionais de saúde estejam preparados para realizar a abordagem aos indivíduos tabagistas durante o atendimento que realizam em suas rotinas de trabalho, conforme preconizado nacional e internacionalmente ${ }^{(13-15)}$.

Estudos destacam, ainda, que o tabagista que recebe aconselhamento para deixar de fumar por diferentes categorias profissionais tem maiores chances de alcançar e de permanecer em abstinência, em decorrência daquele que é atendido por apenas um profissional de saúde ${ }^{(16)}$.

Acreditamos ser de grande relevância a discussão sobre a inclusão da temática do tabagismo na grade curricular acadêmica dos cursos da área da saúde, a fim de melhor qualificar os futuros profissionais no atendimento aos tabagistas, uma vez que este serviço ainda se encontra em processo de estruturação na atenção básica na rede do SUS.

\section{REFERÊNCIAS}

1. U. S. Surgeon General. The Health Consequences of Smoking Nicotine Addiction. Rockville (USA); 1988.

2. World Health Organization. Tabacco or health programme: Guidelines for controlling and monitoring the tabacco. Geneve; 1996.

3. Cavalcante TM. O médico e suas representações sobre tabagismo, fumante e cessação de fumar [dissertação]. Rio de Janeiro (RJ): Escola Nacional de Saúde Pública. Fundação Oswaldo Cruz; 2001.

4. Campos HS. Tabagismo entre os médicos no Brasil. J Epidemiol. 1992 Set/Out; 18(1):325-32.

5. Costa NR. Políticas públicas, justiça distributiva e inovação: saúde e saneamento na agenda social. São Paulo: HUCITEC; 1998.

6. Menezes A, Palma E, Holthausen R, Oliveira R, Oliveira P, Devens E, et al. Evolução temporal do tabagismo em estudantes de medicina, 1986, 1991, 1996. Rev Saúde
Públ. 2001;35(2):165-69.

7. World Health Organization. Who European Strategy for Smoking. Copenhagen (DK); 2004.

8. Mackay J, Eriken M. The tobacco atlas. Geneva. World Health Organization; 2002.

9. Cavalcante JS. O impacto mundial do tabagismo. São Paulo: REALCE; 2002.

10. Rosemberg J. Pandemia do tabagismo: enfoques históricos e atuais. São Paulo: S.E.S; 2002.

11. Allen MB. Medical student's knowledge of smoking. Thorax. 1999;(54):2.

12. Richmond R. Teaching medical students about tobacco. Thorax. 1999;(54):70-8.

13. Ministério da Saúde (BR). Coordenação de prevenção e vigilância. Abordagem e tratamento do fumante Consenso. Rio de Janeiro: INCA; 2001.

14. Machado VC, Alerico MI, Sena J. Programa de prevenção e tratamento do tabagismo: uma vivência acadêmica de enfermagem. Cogitare Enferm. 2007;12(2):248-52.

15. Departament of Health and Human Services (USA). Treating tobacco use and dependence. Rockville: Public Health Service; 2000.

16. Rajamäki H, Katajavuori N, Järvinen P, Hakuli T, Teräsalmi E, Pietilä K. A qualitative study of the difficulties of smoking cessation: health care professionals' and smokers' points of view. Pharm world Sci. 2002;24(6):240-46. 